\title{
Peroral endoscopic myotomy using FlushKnife BT: a single-center series
}

\section{다 $(9)$}

\section{Authors}

Shinwa Tanaka ${ }^{1}$, Takashi Toyonaga ${ }^{2}$, Fumiaki Kawara², Ian S. Grimm³ ${ }^{3}$ Namiko Hoshi ${ }^{1}$, Hirofumi Abe ${ }^{1}$, Yoshiko Ohara ${ }^{1}$, Yoshinori Morita', Eiji Umegaki ${ }^{1}$, Takeshi Azuma ${ }^{1}$

Institutions

1 Division of Gastroenterology, Department of Internal Medicine, Graduate School of Medicine, Kobe University, Kobe, Japan

2 Department of Endoscopy, Kobe University Hospital, Kobe, Japan

3 Division of Gastroenterology and Hepatology, The University of North Carolina at Chapel Hill, Chapel Hill, North Carolina, United States

submitted 29.11.2016

accepted after revision $\quad$ 10.4.2017

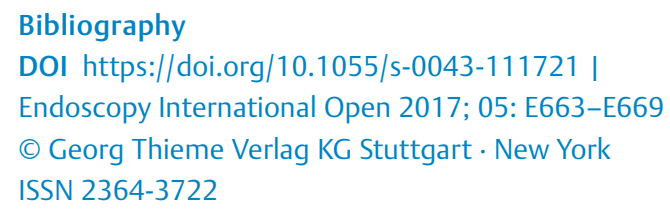

Corresponding author

Takashi Toyonaga, MD, Department of Endoscopy, Kobe University Hospital, 7-5-1 Chu-o-ku, Kusunoki-Cho, Kobe, Hyogo 650-0017, Japan

Fax: +81-78-3826309

toyonaga@bf7.so-net.ne.jp

\section{ABSTRACT}

Background and study aims Peroral endoscopic myotomy (POEM) is an evolving new treatment strategy for achalasia. Although several kinds of electrosurgical knives have been used in performing POEM, the best device has yet to be determined. The FlushKnife BT is a waterjet-emitting short needle-knife with a small ball tip (BT) that offers the potential to perform all aspects of POEM with a single device. In this study, we evaluated the safety and efficiency of the FlushKnife BT for POEM.

Patients and methods A total of 54 consecutive patients with achalasia and other spastic esophageal motility disorders, such as jackhammer esophagus or distal esophageal spasm, who underwent POEM between January 2016 and August 2016, were included in this retrospective study.

Results The median operation time was 73.0 minutes (range 39-184 minutes). All procedures were completed using only the FlushKnife BT without changing to any other electrosurgical instrument. The median number of additional submucosal injections with an injection needle was 0 (range $0-1$ ). Endoscopic vessel sealing was performed a mean of 3 times (range $0-7$ ). The median number of bleeding episodes requiring treatment with hemostatic forceps was 0 (range $0-5$ ). There were no significant adverse events. Seven of 52 patients (13.5\%) reported symptoms of gastroesophageal reflux disease such as heartburn or acid reflux at 3 month follow-up.

Conclusions The FlushKnife BT enabled POEM to be performed with very few device exchanges, either for re-injection or to control intraoperative bleeding. In this uncontrolled case series, the ability of the FlushKnife BT to perform nearly all aspects of the POEM procedure seems to make it particularly well suited to this procedure.

\section{Introduction}

Peroral endoscopic myotomy (POEM) is a recently developed treatment strategy for patients with achalasia [1-3]. To date, a number of studies have demonstrated that POEM can achieve an $82 \%-100 \%$ success rate for palliation of dysphagia [4]. However, relatively few medical centers provide this procedure because of the technical expertise required, and because laparoscopic Heller myotomy and pneumatic dilation remain effective, time-honored therapeutic options.
On the other hand, endoscopic submucosal dissection (ESD) has been widely accepted as a standard treatment for early gastrointestinal neoplasia, when the risk of lymph node metastasis is very low [5-7], especially in Asia. This procedure is now being performed around the world, and a variety of electrosurgical knives have been developed to improve the efficiency and safety of ESD [8-12]. POEM requires techniques such as mucosal incision, submucosal tunneling, hemostasis, and myotomy, which are derived from the basic methods used in performing ESD [3]. 
Not surprisingly, several electrosurgical knives that were initially developed for ESD have since been applied to POEM. Although the use of several kinds of electrosurgical knives in POEM has been reported $[3,13,14]$, the best device has yet to be determined, with the choice of knife often depending on the endoscopist's experience, expertise, and preference. According to previous reports, the triangle tip knife (KD-640L; Olympus Medical Systems, Japan) is most commonly used for POEM, and the HybridKnife (Erbe Elektromedizin $\mathrm{GmbH}$, Tübingen, Germany), which has waterjet function, is also widely used [3, 12]. In general, needle-type electrosurgical knives are preferred in esophageal ESD, given the thin wall of the esophagus and the narrow working space. The FlushKnife BT (DK2618JB; Fujifilm, Tokyo, Japan) is a waterjet-emitting, short needle-knife that is equipped with a spherical tip $0.9 \mathrm{~mm}$ in diameter, for use not only in esophageal ESD but also gastric or colorectal ESD. Because of the small, short ball tip of the FlushKnife BT and its built in waterjet function [15-17], this device offers the potential to perform POEM more efficiently and safely than other ESD knives; however, there have been no reports regarding the use of FlushKnife BT to perform POEM. The aim of this study was to evaluate the feasibility and efficiency of the FlushKnife BT for POEM.

\section{Patients and methods}

\section{Study design}

The study was performed at Kobe University Hospital, a tertiary referral center in Japan. The data for POEM procedures were collected prospectively. Enrollment began in January 2016, and consecutive patients with achalasia and other spastic esophageal motility disorders such as jackhammer esophagus or distal esophageal spasm who were candidates for POEM were recruited for this retrospective study. The current study was approved by the Institutional Review Board. Written informed consent was obtained from all participants, and the study was conducted according to the Declaration of Helsinki.

\section{FlushKnife BT}

The FlushKnife BT is equipped with a spherical tip $0.9 \mathrm{~mm}$ in diameter and comes in four lengths: 1.5, 2, 2.5, and $3 \mathrm{~mm}$ ( $\mathbf{F i g . 1 )}$. A waterjet emitted from the tip of the sheath enables lavage of the operating field, as well as submucosal fluid injection without device exchange [16]. Generally, the $2.5 \mathrm{~mm}$ and 2 $\mathrm{mm}$ long types are used for the stomach, and the $1.5 \mathrm{~mm}$ type is used for ESD in the esophagus and colorectum $[16,18,19]$. The $3 \mathrm{~mm}$ long type was selected for POEM in order to achieve more effective myotomy using the longer arm of the knife ( Fig. 1). The bulbous spherical tip is used to hook onto circular muscle fibers and to provide gentle traction, prior to coagulation and cutting. The settings for FlushKnife BT using the Erbe Vio 300 generator were as follows: spray coagulation mode, effect $1,100 \mathrm{~W}$ was used for creating a submucosal tunnel; endocut I mode, effect 4, duration 3, interval 3, was used for mucosal incision and myotomy.

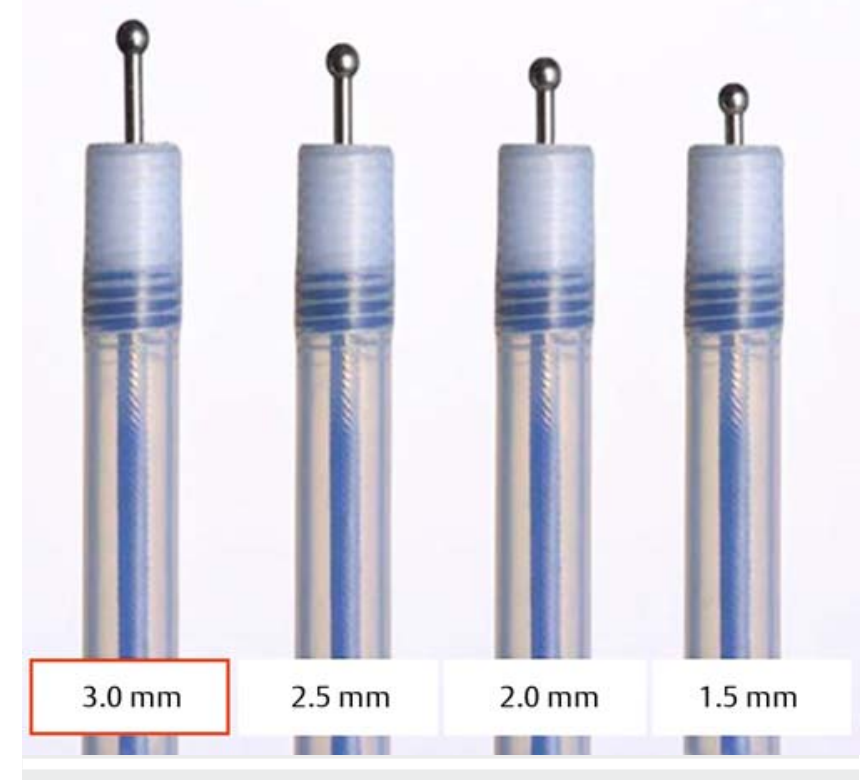

Fig. 1 The FlushKnife BT (Fujifilm, Tokyo, Japan). The $3 \mathrm{~mm}$ long type was selected for peroral endoscopic myotomy.

\section{POEM technique}

A forward-viewing endoscope with a $3.2 \mathrm{~mm}$ instrumentation channel (GIF-H260); Olympus Medical Systems) and a short ST hood (DH-28GR; Fujifilm) were used. POEM was performed under general anesthesia with endotracheal intubation and carbon dioxide insufflation. First, approximately $5 \mathrm{~mL}$ of saline mixed with $0.3 \%$ indigo carmine was injected into the submucosa ( $\triangleright$ Fig. 2a), and a longitudinal mucosal incision was made in the mucosal surface to gain access to the submucosal space. This same saline mixture was used for subsequent injections via the FlushKnife BT. Second, a submucosal tunnel was created and extended past the esophagogastric junction (EGJ) for $2-3 \mathrm{~cm}$ into the gastric cardia ( $\triangleright \mathbf{F i g} \cdot \mathbf{2 b}, \mathbf{c}$ ). The submucosal tunnel was usually created in the 5 o'clock position, or the 7 o'clock position for patients with a history of prior Heller myotomy in order to avoid fibrosis from the previous surgery. Complete gastric myotomy was confirmed by using a second, smallcaliber endoscope [20]. Third, a proximal-to-distal circular myotomy was performed, with care, to preserve the longitudinal muscle layers of the esophagus and stomach ( $\triangleright \mathbf{F i g .} \mathbf{2} \mathbf{d , e}$ ). The myotomy was extended $2-3 \mathrm{~cm}$ into the gastric cardia beyond the EG] in patients with achalasia and in those with other spastic esophageal motility disorders. Finally, the mucosal entry was closed using endoscopic clips ( $\triangleright$ Fig. $2 \mathbf{f}$ ).

\section{Precoagulation}

When small-to-medium-sized (less than $2 \mathrm{~mm}$ ) blood vessels were encountered during POEM, in endoscopic vessel sealing technique was performed, as described previously [21]. The procedure is performed as follows. First, submucosal layer tissue surrounding a blood vessel was dissected and the blood vessel was isolated ( $\mathbf{F i g . 3 a ) . ~ S e c o n d , ~ b o t h ~ s i d e s ~ o f ~ t h e ~}$ isolated blood vessel were compressed with the tip of the 


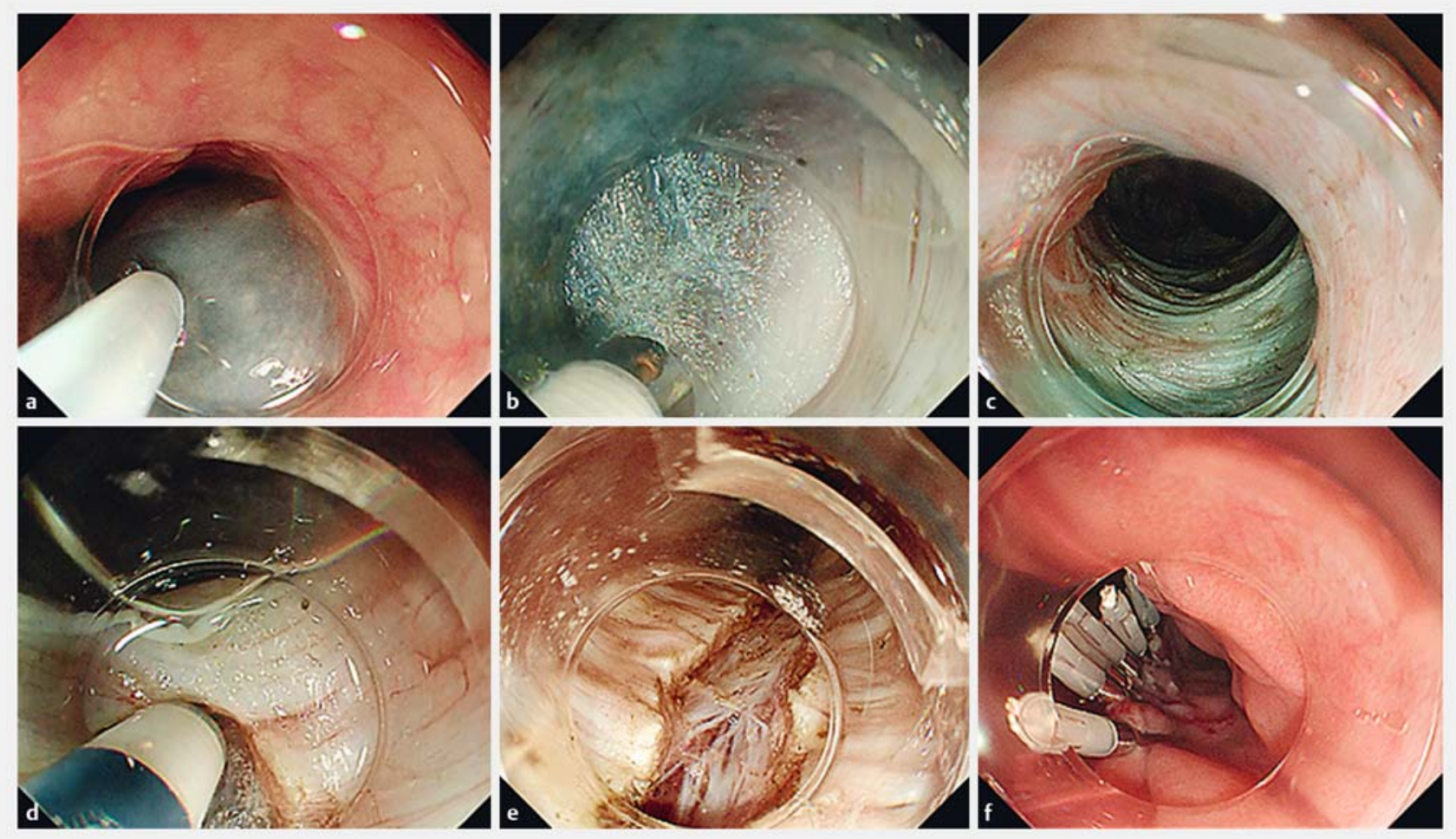

- Fig. 2 Peroral endoscopic myotomy using the FlushKnife BT (Fujifilm, Tokyo, Japan). a A needle-knife was used for mucosal injection. b The FlushKnife BT was used to create a submucosal tunnel. c After creation of the submucosal tunnel. $\mathbf{d}$ The FlushKnife BT was used to cut the circular muscle. e The longitudinal muscle remained intact after myotomy. $\mathbf{f}$ The mucosal entry was closed using endoscopic clips.
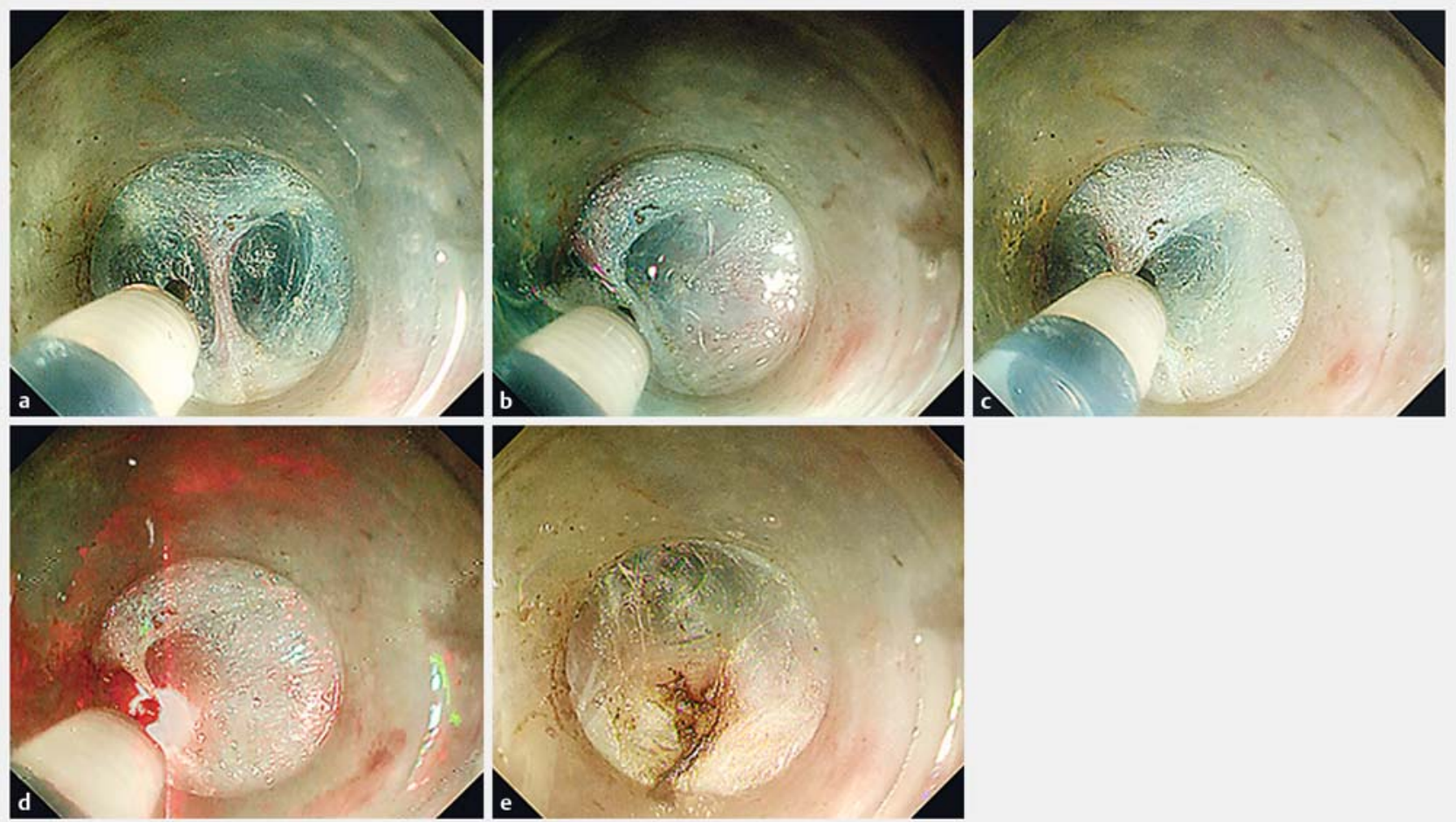

Fig. 3 Endoscopic vessel sealing technique using the FlushKnife BT (Fujifilm, Tokyo, Japan). a The blood vessel in the submucosal layer was isolated. b, c Both sides of the isolated blood vessel were clamped using the tip of the FlushKnife BT, and then precoagulated in forced coagulation mode (effect $1,10 \mathrm{~W}$ ) until the vessel turned white. $\mathbf{d}$ The blood vessel was dissected in the spray coagulation mode (effect $1,100 \mathrm{~W}$ ). e After dissection of the blood vessel. 
- Table 1 Patient demographics and perioperative characteristics.

\begin{tabular}{|c|c|}
\hline & $n=54$ \\
\hline Age, median (IQR), [range], years & $52.5(43-66),[16-84]$ \\
\hline \multicolumn{2}{|l|}{ Sex } \\
\hline - Men & 24 \\
\hline - Women & 30 \\
\hline BMI, median (IQR), [range], $\mathrm{kg} / \mathrm{m}^{2}$ & $20.6(19.0-22.2),[14.1-31.6]$ \\
\hline $\begin{array}{l}\text { Duration of symptoms, median } \\
\text { (IQR), [range], years }\end{array}$ & $4(1.5-15),[0.5-60]$ \\
\hline \multicolumn{2}{|l|}{ Type of achalasia, n } \\
\hline - Straight type & 39 \\
\hline - Sigmoid type & 11 \\
\hline \multicolumn{2}{|l|}{ Chicago classification, $\mathrm{n}$} \\
\hline - Type I & 22 \\
\hline - Type II & 21 \\
\hline - Type III & 3 \\
\hline " Jackhammer esophagus & 3 \\
\hline - Distal esophageal spasm & 1 \\
\hline - Unknown & 4 \\
\hline \multicolumn{2}{|l|}{ Primary procedure, $\mathrm{n}$} \\
\hline - Balloon dilation & 11 \\
\hline - Heller myotomy & 2 \\
\hline $\begin{array}{l}\text { Myotomy length, median (IQR), } \\
\text { [range], cm }\end{array}$ & $13(10.3-16),[4-23]$ \\
\hline Submucosal fibrosis, $\mathrm{n}$ & 6 \\
\hline IQR, interquartile range; BMI, body ma & ndex. \\
\hline
\end{tabular}

FlushKnife BT and precoagulated in low power, forced coagulation mode (Effect 1, 10 W) until the blood vessel turned white ( $>$ Fig.3b,c). Finally, the blood vessel was dissected using the spray coagulation mode (effect 1, 100 W) ( Fig. 3d,e).

\section{Statistical analysis}

Medians (interquartile range [IQR]) and percentages for categorical variables were used to describe patient baseline characteristics and procedure-related parameters.

Eckardt scores and integrated relaxation pressures (IRP) before and after POEM were compared, and changes in Eckardt score and IRP at 3 months after the POEM procedure were assessed using Wilcoxon's signed rank sum test.

\section{Results}

\section{Clinical features of patients}

POEM was carried out in 54 patients between January 2016 and August 2016. - Table 1 lists the patient demographic and perioperative characteristics. Of the 54 patients, including 24 men and 30 women, the median age was 52.5 years (range 16-84 years). Median body mass index was $20.6 \mathrm{~kg} / \mathrm{m}^{2}$ (range 14.1 $31.6 \mathrm{~kg} / \mathrm{m}^{2}$ ). Median duration of symptoms was 4 years (range $0.5-60$ years). Esophageal manometry findings were classified according to the Chicago classification system, as type I (22 patients, $40.7 \%$ ), type II (21 patients, 38.9\%), type III (3 patients, $5.6 \%$ ), jackhammer esophagus (3 patients, 5.6\%), distal esophageal spasm (1 patient, $1.9 \%$ ). In four patients, manometry was not completed because of difficulty in inserting the catheter through the lower esophageal sphincter. Among the 50 achalasia patients, 39 (78.0\%) were classified as straight type and 11 (22.0\%) as sigmoid type. Prior to POEM, pneumatic balloon dilation had been performed in 11 patients (20.4\%) and 2 patients (3.7\%) had undergone Heller myotomy.

The median total length of the endoscopic myotomy was $13.0 \mathrm{~cm}$ (range $4-23 \mathrm{~cm}$ ). Submucosal fibrosis was confirmed in six patients $(11.1 \%)$.

\section{Procedure-related outcomes}

- Table 2 shows the procedure-related outcomes. The median operating time was 73.0 minutes (range 39-184 minutes). All procedures were completed using only the FlushKnife BT without exchange to another electrosurgical device. The median number of additional submucosal injections requiring an injection needle was 0 (range $0-1$ ). The median number of bleeding

- Table 2 Procedure-related outcomes.

Operation time, median (IQR), [range], minutes

$73(55.3-91),[39-184]$

Endoknife changes, median (IQR), [range], $\mathrm{n}$

$0(0),[0]$

Additional submucosal injection with injection needle, median (IQR), [range], n

$0(0),[0-1]$

Bleeding requiring hemostatic forceps, median (IQR), [range], n

$0(0-1.8),[0-5]$

Number of times vessel sealing required during procedure, median (IQR), [range], $n$

$3(1-4),[0-7]$

Complications, $\mathrm{n}(\%)$

- Pneumoperitoneum requiring intervention

$5(9.3)$

- Mucosal perforation

$3(5.6)$

$I Q R$, interquartile range. 


\begin{tabular}{|c|c|c|c|}
\hline & Before POEM & 3 months after POEM & $P$ value \\
\hline Eckardt score, median (IQR), [range] & $6(4-7),[2-11]$ & $0(0-1),[0-4]$ & $<0.001$ \\
\hline IRP, median (IQR), [range], mmHg & $26(18.9-33.5),[2.3-75]$ & $9(5.9-11.9),[0.1-23.8]$ & $<0.001$ \\
\hline Endoscopic reflex esophagitis findings, n (\%) & & $\begin{array}{l}\text { Grade N: } 16(30.8) \\
\text { Grade A: } 17(32.7) \\
\text { Grade B: } 15(28.8) \\
\text { Grade C: } 3(5.8) \\
\text { Grade D: } 1(1.9)\end{array}$ & \\
\hline GERD symptoms, n (\%) & & $7(13.5)$ & \\
\hline
\end{tabular}
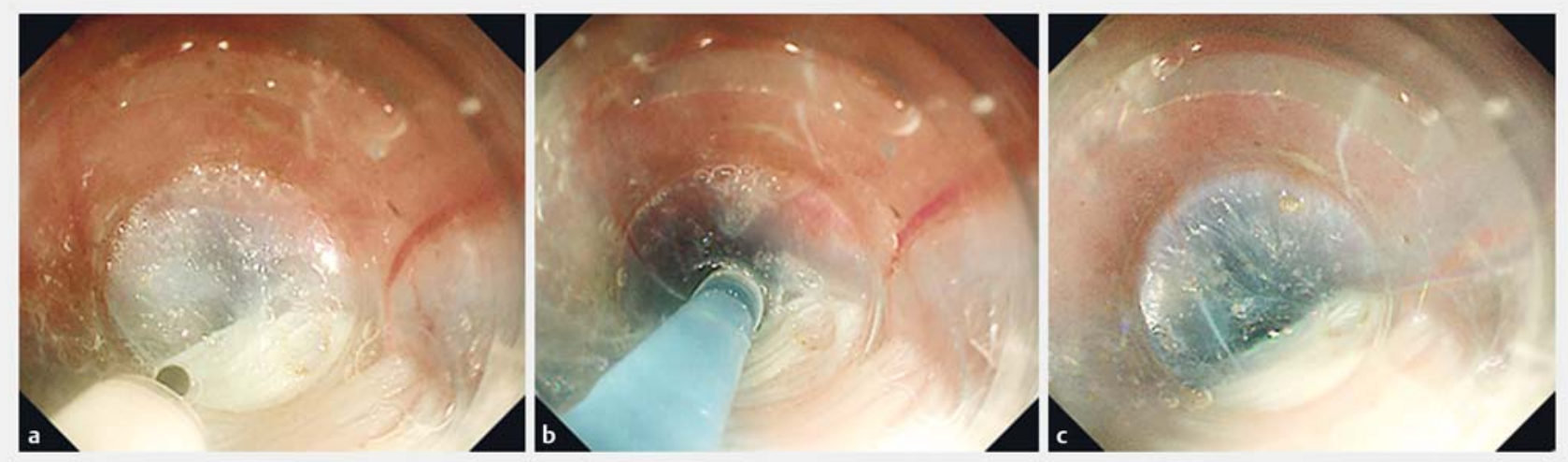

- Fig. 4 Submucosal injection using the FlushKnife BT (Fujifilm, Tokyo, Japan). a The submucosal space is very tight at the esophagogastric junction. b Submucosal injection using the FlushKnife BT. c Enough space was thus created to allow safe passage through to the gastric side.

episodes requiring treatment with hemostatic forceps was 0 (range $0-5$ ). Endoscopic vessel sealing was performed a median of 3 times per POEM procedure (range $0-7$ ).

\section{Treatment outcomes}

Esophageal manometry, upper endoscopy, and interview (Eckardt score, symptoms of gastroesophageal reflux disease [GERD]) were performed in all patients, 3 months following POEM. Two patients did not receive follow-up examinations for personal reason. - Table 3 shows the treatment outcomes. A significant reduction in symptoms was achieved (median [range], preoperative Eckardt score 6 [2-11] vs. postoperative $0[0-4] ; P<0.001$ ), with a significant reduction in IRP (median [range], preoperative $26.0 \mathrm{mmHg}$ [2.3-75] vs. postoperative $9 \mathrm{mmHg}[0.1-23.8] ; \quad P<0.001)$. Thirty-six of 52 patients $(69.2 \%)$ had endoscopic findings of reflux esophagitis. Seven of 52 patients (13.5\%) complained of GERD symptoms such as heartburn or acid reflux.

\section{Discussion}

Our results of POEM with the FlushKnife were similar to those in other published series. Our median operation time was 73.0 minutes (range 39-184 minutes) whereas previous studies re- ported a range of 22.9-148 minutes [22-25]. We observed a significant reduction in symptoms: (median [range], preoperative Eckardt score $6[2-11]$ vs. postoperative 0 [0-4]; $P<$ 0.001 ), with a significant reduction in IRP (median [range], preoperative $26.0 \mathrm{mmHg}[2.3-75]$ vs. postoperative $9 \mathrm{mmHg}$ [0.1-23.8]). Seven of 52 patients $(13.5 \%)$ complained of GERD symptoms such as heartburn or acid reflux, which is also comparable to that reported in published literature [22-25]. No patient experienced a serious adverse event.

We consider the primary benefit of the FlushKnife BT to be the need for fewer device exchanges during the procedure, which we attribute both to its waterjet function and to its good hemostatic capability [16]. In our study, the number of knife exchanges, additional submucosal injections with an injection needle, and episodes of bleeding requiring hemostatic forceps were very few. This enabled endoscopists to perform their procedure continuously, without interruption. Waterjet function effectively provided submucosal injection without requiring re-introduction of an injection needle. Even the initial submucosal injection can occasionally be performed by the FlushKnife itself, instead of an injection needle. In this study, submucosal injection using an injection needle was performed only once in one patient. Waterjet irrigation via the FlushKnife is especially useful when passing through a tight EGJ in a pa- 
tient with achalasia during submucosal tunnel creation. Repeated submucosal injection is often required to maintain enough space to allow safe passage through to the gastric side ( $\triangleright$ Fig.4). However, the risk of mediastinitis due to the leakage of liquid into the mediastinum is a concern. To reduce the risk of mediastinitis, it is better to use the waterjet function as little as possible during myotomy.

Intraoperative bleeding is a serious problem that can complicate POEM. Prevention of bleeding is important not only for the hemodynamic stability of the patient, but also to maintain a clear operative field. If bleeding develops within the submucosal tunnel, the submucosal layer is easily obscured by blood and/or excessive charring, and the rate of mucosal perforation or further bleeding may consequently be increased. To prevent bleeding, it is important to identify and isolate blood vessels and precoagulate them before transection [26]. Theoretically, the current density in the FlushKnife BT is decreased at its spherical tip. This provides better hemostatic capability, allowing for small vessels to be precoagulated and cut by the knife itself [16]. Furthermore, the endoscopic vessel sealing technique using FlushKnife BT was easy and effective for coagulation of small-to-medium-sized vessels without the need for hemostatic forceps [21]. This technique was frequently employed during POEM, and contributed to a reduction of bleeding episodes that required hemostatic forceps, as well as maintaining a clear operative field.

Another technical challenge encountered during POEM is the management of fibrotic areas [27]. In this study, six patients had submucosal fibrosis. Even in these patients, however, POEM could be completed with the FlushKnife BT alone, without changing to another electrosurgical device. Previous literature indicates that the FlushKnife BT is effective for overcoming submucosal fibrosis in ESD $[17,28]$. To dissect a severely fibrotic area, it is important to proceed by $1-2 \mathrm{~mm}$ increments, and to start in an area that is least fibrotic. This approach enables clear exposure of new fibrotic areas, little by little, and helps to identify an appropriate line of dissection through a severely fibrotic area. If dissection using spray coagulation mode is difficult, the change to swift coagulation mode or cut mode will enable sharp cutting.

Although the FlushKnife BT has several advantages as indicated above, there is also a disadvantage. The tip of the FlushKnife BT is small, which enables very precise manipulation; however, compared with the triangle tip knife, it is less efficient at creating the submucosal tunnel, and this stage can become time-consuming in patients requiring a long myotomy.

In conclusion, the FlushKnife BT enabled effective POEM with very few device changes, and with minimal need for additional submucosal injections or use of the hemostatic forceps. The ability of the FlushKnife BT to efficiently perform nearly all aspects of the POEM procedure makes it particularly well suited to this procedure. To determine the relative safety, efficacy, and potential cost savings associated with the use of FlushKnife BT for POEM, compared with other available electrosurgical knives, a multicenter, randomized controlled study should be considered.

\section{Competing interests}

\section{None}

References

[1] Ngamruengphong S, Inoue H, Chiu P et al. Long-term outcomes of per-oral endoscopic myotomy in achalasia patients with a minimum follow-up of 2 years: an international multicenter study. Gastrointest Endosc 2017; 85: 927 - 933

[2] Bechara R, Onimaru M, Ikeda H et al. Per-oral endoscopic myotomy, 1000 cases later: pearls, pitfalls, and practical considerations. Gastrointest Endosc 2016; 84: 330 - 338

[3] Inoue $\mathrm{H}$, Minami $\mathrm{H}$, Kobayashi Y et al. Peroral endoscopic myotomy (POEM) for esophageal achalasia. Endoscopy 2010; 42: 265-271

[4] NOSCAR POEM White Paper Committee, Stavropoulos SN, Desilets D] et al. Peroral endoscopic myotomy white paper summary. Gastrointest Endosc 2014; 80: 1 - 15

[5] Rembacken B], Gotoda T, Fujii T et al. Endoscopic mucosal resection. Endoscopy 2001; 33: 709-718

[6] Soetikno R, Gotoda T, Nakanishi Y et al. Endoscopic mucosal resection. Gastrointest Endosc 2003; 57: $567-579$

[7] Soetikno R, Kaltenbach T, Yeh R et al. Endoscopic mucosal resection for early cancers of the upper gastrointestinal tract. J Clin Oncol 2005; 23: $4490-4498$

[8] Yano T, Ono H, Doi T et al. Endoscopic submucosal dissection using a new scissors-type electrosurgical knife: a first-in-human feasibility study. Endoscopy 2014; 46: $754-757$

[9] Kanzaki H, Ishihara R, Ohta T et al. Randomized study of two endoknives for endoscopic submucosal dissection of esophageal cancer. Am J Gastroenterol 2013; 108: $1293-1298$

[10] Fujinami H, Hosokawa A, Ogawa K et al. Endoscopic submucosal dissection for superficial esophageal neoplasms using the stag beetle knife. Dis Esophagus 2014; 27: 50 - 54

[11] Homma K, Otaki Y, Sugawara M et al. Efficacy of novel SB knife Jr examined in a multicenter study on colorectal endoscopic submucosal dissection. Dig Endosc 2012; 24: (Suppl. 01): 117-120

[12] Schumacher B, Charton JP, Nordmann T et al. Endoscopic submucosal dissection of early gastric neoplasia with a water jet-assisted knife: a Western, single-center experience. Gastrointest Endosc 2012; 75: $1166-1174$

[13] Tang X, Gong W, Deng Z et al. Comparison of conventional versus Hybrid knife peroral endoscopic myotomy methods for esophageal achalasia: a case-control study. Scand J Gastroenterol 2016; 51: 494 500

[14] Minami H, Isomoto H, Yamaguchi N et al. Peroral endoscopic myotomy for esophageal achalasia: clinical impact of 28 cases. Dig Endosc 2014; $26: 43-51$

[15] Toyonaga T, Man-i M, Chinzei R et al. Endoscopic treatment for early stage colorectal tumors: the comparison between EMR with small incision, simplified ESD, and ESD using the standard flush knife and the ball tipped flush knife. Acta Chir lugosl 2010; 57: 41-46

[16] Toyonaga T, Man-I M, Fujita T et al. The performance of a novel balltipped Flush knife for endoscopic submucosal dissection: a case-control study. Aliment Pharmacol Ther 2010; 32: 908 - 915

[17] Toyonaga T, Nishino E, Man-I M et al. Principles of quality controlled endoscopic submucosal dissection with appropriate dissection level and high quality resected specimen. Clin Endosc 2012; 45: 362 - 374

[18] Tanaka S, Toyonaga T, Morita Y et al. Efficacy of a new hemostatic forceps during Gastric endoscopic submucosal dissection: a prospec- 
tive randomized controlled trial. J Gastroenterol Hepatol 2017; 32: $846-851$

[19] Tanaka S, Toyonaga T, Morita Y et al. Feasibility and safety of endoscopic submucosal dissection for lower rectal tumors with haemorrhoids. World J Gastroenterol 2016; 21: 6268-6275

[20] Grimes KL, Inoue H, Onimaru M et al. Double-scope per oral endoscopic myotomy (POEM): a prospective randomized controlled trial. Surg Endosc 2016; 30: $1344-1351$

[21] Tanaka S, Toyonaga T, Morita Y. Endoscopic vessel sealing: a novel endoscopic precoagulation technique for blood vessels during endoscopic submucosal dissection. Dig Endosc 2013; 25: $341-342$

[22] Von Renteln D, Fuchs KH, Fockens P et al. Peroral endoscopic myotomy for the treatment of achalasia: an international prospective multicenter study. Gastroenterology 2013; 145: 309-311

[23] Shiwaku H, Inoue H, Yamashita K et al. Peroral endoscopic myotomy for esophageal achalasia: outcomes of the first over 100 patients with short-term follow-up. Surg Endosc 2016; 30: 4817-4826
[24] Inoue H, Sato H, Ikeda H et al. Per-oral endoscopic myotomy: a Series of 500 patients. J Am Coll Surg 2015; 221: 256-264

[25] Cai MY, Zhou PH, Yao LQ et al. Peroral endoscopic myotomy for idiopathic achalasia: randomized comparison of water-jet assisted versus conventional dissection technique. Surg Endosc 2014; 28: 1158 1165

[26] Toyonaga T, Man-I M, Fujita T et al. Endoscopic submucosal dissection using the Flush knife and the Flush knife BT. Tech Gastrointest Endosc 2011; 13: 84-90

[27] Teitelbaum EN, Soper NJ, Arafat FO et al. Analysis of a learning curve and predictors of intraoperative difficulty for peroral esophageal myotomy (POEM). J Gastrointest Surg 2014; 18: 92 - 98

[28] Ishida T, Jacob H, Toyonaga T. The goals and pitfalls of gastric submucosal dissection: a special focus on dissection of lesions with severe fibrosis. Korean J Helicobacter Up Gastrointest Res 2014; 14: $163-173$ 